\title{
Science, Médecine et Homéopathie: essai sur les critères de compréhension réciproque
}

Guy Loutan

Correspondance:

Dr Guy Loutan

Médecin praticien FMH, psychosomatique AMPP, homéopathie FMH

4 Bis, rte de Jussy

CH-1226 Genève Thônex

loutan.guy[at]bluewin.ch
Nos contacts entre médecins allopathes et homéopathes semblent manquer de références communes. Aussi de distance par rapport aux a priori et poncifs: «il n'y a rien dans vos remèdes», «vous empoisonnez les patients avec votre chimie»... Abandonnons la dispute traditionnelle pour reprendre la discussion sereinement..

Pour nous comprendre, au moment de la (ré)entrée de certaines thérapies complémentaires dans la LAMal, tentons de

A Nous entendre sur le sens donné aux mots «scientifique» et «science», et contrôlons s'ils jouissent vraiment d'un brevet d'usage exclusif.

B Puis abordons quelques visions philosophiques et anthropologiques sous lesquels nous envisageons l'homme [1].

C Enfin, à partir de ces bases, discutons avec l'humilité des scientifiques et le respect mutuel des philosophes.

\section{A: science et scientifique}

Le mot «science» [2] concerne généralement pour la faculté «officielle», l'étude du pondéral, mesurable, chiffrable, visible. Pourtant le mot «science» ne peutil / ne doit-il pas être pris dans des acceptions tout aussi sérieuses et plus ouvertes? Si oui, lesquelles, si non, pourquoi?

Science

- Somme de connaissances qu'un individu possède ou peut acquérir par l'étude, la réflexion ou l'expérience.

- Avoir sur quelque chose des informations sûres que l'on ne peut mettre en doute.

- Ensemble de connaissances systématisées se rapportant à un même domaine.

- Ensemble des connaissances humaines systématisées relevant de telle méthode ou de telle visée spécifique. (Science expérimentale, matérialiste, pure; science d'observation.)

Scientifique

- Qui révèle le savoir-faire de quelqu'un.

- Qui porte sur un sujet relevant de la / d'une science et est le résultat d'une activité menée dans ce domaine.

- Qui utilise à des fins propres les méthodes et les acquis de la science (correspondante, nda).

\section{B: visions philosophiques et anthropologiques}

Toute thérapie se base sur des paradigmes, une philosophie et une anthropologie spécifiques. Les références de chacun à ces branches de la pensée qui en- cadrent les sciences correspondant à ces thérapies doivent être annoncées en début de discussion.

Philosopher ne signifie pas élucubrer sur des convictions personnelles, mais chercher à connaitre rationnellement, par l'expérience et l'usage de nos sens, la vérité, les principes et les fins des choses, comme dans la philosophie naturelle [3], excluant tout ce qui est du domaine de la révélation.

La recherche dans chaque type de thérapie va impliquer le développement d'une vision correspondante du bénéficiaire, tout comme la science correspondante amène à des remèdes adaptés au mode de pensée utilisé.

\section{Exemples}

1. L'homme est un être énergétique, système complexe de réseaux de trajets énergétiques, (méridiens en acupuncture): la thérapie adéquate doit harmoniser, réguler ces circuits pour supprimer un symptôme ou rendre la santé au patient, selon la profondeur du traitement. Le paradigme scientifique est «signal énergétique».

2. L'homme est un ensemble ou une addition de réactions physicochimiques étudiables, quantifiables, séparables les unes des autres [4]: la thérapie adéquate est analytique, mécaniste et travaille avec des remèdes efficaces sur des paramètres physicochimiques. Selon des paradigmes quantitatifs, elle touche des réactions biologiques mesurables, ponctuelles et ciblées, selon une vision cause-effet souhaitée linéaire. Ce morcellement est logique dans cette approche, et indispensable à la recherche et au développement des médicaments physicochimiques.

Le patient est «guéri» d'un symptôme, d'un diagnostic. Les statistiques peuvent démontrer l'effet d'un remède sur un symptôme tout en excluant de l'étude les éventuelles modifications des autres. Les effets biologiques, microbiologiques, radiologiques et autres sont objectivables, chiffrables, pondérables, d'où le terme de thérapie objectivante donnée à l'allopathie [5].

3. L'homme est un être culturel abordé par le langage: la thérapie est verbale, comme en psychanalyse. La thérapie est dirigée sur un aspect du patient, mesurable par son récit, sans possibilité de mesure objective du résultat (quoi que, avec l'imagerie moderne...), si ce n'est par l'estimation de son confort psychique et donc subjectif.

4. Enfin, l'homme est un composé substantiel [6] comme dénommé en anthropologie aristotélicothomiste, un tout complexe. 


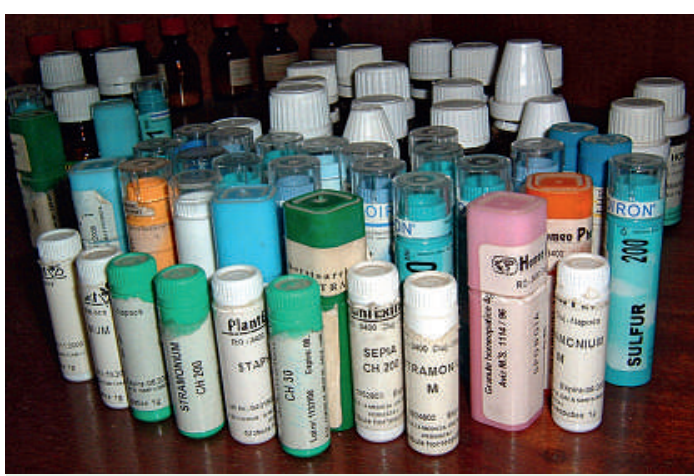

L'homéopathie uniciste veut aborder tant le médicament que le patient comme un tout.

Aristote? Parce qu'il a développé ce qu'on appelle la philosophie naturelle, étude et connaissance de ce qui est, abordable par les sens, sans a priori limitatif.

Thomiste parce que Thomas d'Aquin a accepté la notion de finalité des parties par rapport au tout, et surtout une finalité humaine: amour, créativité, connaissance, spiritualité... qui dépassent les finalités des fonctions vitales biologiques de nutrition, croissance, reproduction.

Cette optique nécessite une thérapie qui touche tout le système, sa finalité comme celle de chacune de ses parties. Cette thérapie ne peut être parcellaire, ne peut agir de façon uniquement mesurable, chiffrée, quantitative. On ne pèse ni ne radiographie la vie, la santé ou le bien-être. C'est du vécu, non des choses. L'effet des substances médicinales se découvre par l'expérience sur le vivant, non sur un paramètre isolé. Cette vision implique l'étude d'un système complexe, de toutes les modifications objectives et subjectives, aucune n'étant retranchable du tout sans biaiser l'observation par le reniementmême de l'attitude scientifique adéquate.

L'homéopathie uniciste est la thérapie typique dont la philosophie et l'anthropologie permettent d'aborder tant le médicament que le patient, comme un tout [7]. Car le tout du sujet sensible et réactif est concerné pour percevoir et décrire les effets du médicament lors de l'expérimentation chez le sujet sain (pathogénésie) [8]. Sujet intelligent aussi pour décrire ces effets dans tous les domaines lors de la maladie à traiter.

L'homéopathie entre dans les thérapies analogiques [9], puisque basée sur l'étude des rapports de ressemblance entre des objets différents: les effets expérimentaux d'un remède et les symptômes spontanés d'un patient.

\section{C: I'humilité des scientifiques et le respect mutuel des philosophes}

En conclusion: la scientificité d'une thérapie semble pouvoir être comprise de façon plus vaste que l'acception moderne que notre médecine officielle en a: linéarité, évidence, matière...
La philosophie et l'anthropologie selon laquelle on aborde l'être humain doivent être décrites en préambule pour pouvoir comprendre leur adéquation à tout projet de recherche médicale. Nous devrons donc à partir de ce qui précède nous mettre d'accord sur le type de recherche compatible avec d'un côté la vision de l'homme que l'on veut explorer, et conséquemment accepter de l'autre la démarche intellectuelle adaptée à cette recherche et l'instrument thérapeutique correspondant.

Toute recherche doit être adaptée dans son projet et ses instruments à l'objet étudié, et non l'objet étudié limité aux facettes accessible à une science en l'occurrence inadaptée, même si adéquate à d'autres objets.

L'introduction des thérapies complémentaires dans l'assurance de base implique une redéfinition importante pour chaque thérapie de ses paradigmes et de son anthropologie, ce qui permettra compréhension et respect réciproques. Nous pourrons alors parler confraternellement des thérapies complémentaires.

\section{Références}

1 Conférence 2011 à l'AFADH du Pr. phil. Marcelo Gerstner, Université Catholique Argentine.

2 Définitions du Centre national de ressources textuelles et lexicales (CNRTL). www.cnrtl.fr

3 Naturel: Histoire des idées. Classification, méthode naturelle: fondées sur l'ensemble des caractères, par opposition à la classification, à la méthode artificielle, qui établissent la classification d'après quelques caractères choisis arbitrairement (CNRTL).

4 Bernard C. Introduction à l'étude de la médecine expérimentale;1865: «En biologie, [...] le physiologiste pourra diriger la manifestation des phénomènes de la vie comme le physicien et le chimiste dirigent les phénomènes naturels dont ils ont découvert les lois; mais pour cela l'expérimentateur n'agira pas sur la vie.» (II, ch $1, \S 1$ )

5 Marchat P. L'objet de l'homéopathie: le corps vécu. Ed. Marrimpouey; 2006.

6 Substantiel: qui est en soi, réalité permanente qui sert de support aux accidents, qualités, attributs (couleur, grandeur, forme...). La substance demeure sous le changement.

Composé substantiel: ce qui tout en étant composé de parties est totalement soi et rien d'autre. Georges, tout en étant composé de foie, membres, cerveau, os, sang.. est totalement Georges, il n'est rien d'autre, ni de plus ou de moins que Georges, ni personne d'autre! (CNRTL)

7 Loutan G. L'homéopathie uniciste, instantanés sur une médecine durable. Ed. Loutan; 2010.

8 Hahnemann S. Essai sur un nouveau principe pour découvrir les vertus curatives des substances médicinales. In: Etudes de médecine homéopathique. Paris: Baillières;1850. p. 1796.

9 Effet paradoxal, action-réaction, Arndt-Schulz... Imitons la nature: «qui guérit par l'effet direct du médicament une maladie semblable, car l'effet indirect fait sortir l'organisme de la maladie.» (Hahnemann [8]) 